\title{
Alteraciones posturales de la columna vertebral asociadas al uso de tacones
}

\section{Postural alterations of the spine associated with the use of heels}

\author{
Paulina De Regil-González, ${ }^{*}$ Perla Zuriel Santiago-Galindo, ${ }^{\ddagger}$ Tania Inés Nava-Bringas§
}

\section{RESUMEN}

Introducción: El dolor lumbar es una de las principales causas de atención médica; afecta hasta $80 \%$ de la población mundial en algún momento de su vida. Existen varios factores de riesgo de presentar dolor lumbar o condicionar su persistencia. Durante mucho tiempo se ha postulado la relación del dolor lumbar con la selección del calzado, particularmente con el uso de tacones, esto debido a las alteraciones posturales de la columna vertebral con su uso; sin embargo, no existe un estándar establecido del calzado óptimo ni de la asociación directa del mismo con el dolor lumbar. Objetivo: Analizar la literatura disponible relacionada con las alteraciones posturales de la columna vertebral asociadas al uso de tacones. Material y métodos: Revisión de la literatura disponible en los principales motores de búsqueda en el periodo de 1990 a 2018. Se incluyeron artículos en inglés y español que describieran población con uso de tacones y alteraciones posturales o funcionales de la columna lumbar. Resultados: Se seleccionaron 14 artículos. Estudios previos fundamentan la relación entre zapatos de tacón e incremento de lordosis lumbar y, por consiguiente, dolor lumbar; sin embargo, existe literatura que establece que los zapatos de tacón no afectan la lordosis lumbar en bipedestación, incluso se reporta disminución de la lordosis lumbar con el uso de este tipo de zapatos. Conclusiones: El uso de tacones está relacionado con reducción de la lordosis, retroversión pélvica y ajustes posturales que pueden asociarse a incrementar el estrés en los elementos posteriores del segmento lumbar y secundariamente favorecer el dolor lumbar.

Palabras clave: Tacones, postura, columna lumbar, lordosis, calzado, lumbalgia.

Nivel de evidencia: III

\begin{abstract}
Introduction: Low back pain is one of the main causes of medical care, affecting up to $80 \%$ of the world population at some time in their life. There are several risk factors for presenting low back pain or conditioning its persistence. The relationship of low back pain with footwear selection has been postulated for a long time, particularly with the use of heels, this due to postural alterations of the spine with its use, however there is no established standard of optimal footwear or its direct association with low back pain. Objective: To analyze the available literature related to postural alterations of the spine associated with the use of heels. Material and methods: Review of the literature available in the main search engines with a period from 1990 to 2018. Articles in English and Spanish were included, which included population wearing heels and described postural or functional alterations of the lumbar spine. Results: 14 articles were selected for review. Previous studies support the relationship between high heels and increased lumbar lordosis and, consequently, low back pain, however, there is literature that states that high-heeled shoes do not affect lumbar lordosis in standing, even report a decrease in lumbar lordosis wearing high heels. Conclusions: The use of heels is related to lordosis reduction, pelvic retroversion and postural adjustments that can be associated with increasing stress on the posterior elements of the lumbar segment and secondarily favor low back pain.
\end{abstract}

Keywords: Heels, posture, lumbar spine, lordosis, footwear, low back pain.

Level of evidence: III
* Jefa del Servicio de Rehabilitación del Centro Médico ABC Santa Fe.

‡ Médico Residente de Especialidad de Medicina de Rehabilitación, Instituto Nacional de Rehabilitación "Luis Guillermo Ibarra Ibarra».

$\S$ Rehabilitación de Columna Vertebral, Instituto Nacional de Rehabilitación «Luis Guillermo Ibarra Ibarra».

Recibido para publicación: 29/04/2019. Aceptado: 08/11/2019.

\author{
Correspondencia: \\ Paulina De Regil-González \\ Av. Carlos Graef Fernández Núm. 154, \\ Col. Tlaxala Santa Fe, 05300, Alcaldía Cuajimalpa, \\ Ciudad de México, México. \\ E-mail: pderegilg@abchospital.com \\ dra.deregil@gmail.com
}




\section{INTRODUCCIÓN}

El dolor lumbar es una de las principales causas de atención médica, afecta hasta $80 \%$ de la población en algún momento de su vida. ${ }^{1}$ La gran mayoría de los cuadros de dolor lumbar agudo tienen una resolución progresiva en pocas semanas y se calcula que sólo $10 \%$ de los casos supera las 12 semanas; no obstante, la prevalencia del dolor crónico lumbar no es despreciable, pues alcanza prevalencias de entre 15 y $36 \%$. $^{2}$ Aproximadamente, $30 \%$ de los portadores de lumbalgia en México requieren incapacidad. En el Reino Unido el ausentismo asociado a esta enfermedad es de 13\%, mientras que en la Unión Americana es de 33\%. ${ }^{3}$

Se ha estimado que el costo total del dolor de espalda en los Estados Unidos oscila entre 100,000 y 200,000 millones de dólares (de 119,000 a 238,000 millones de dólares en 2013), con un tercio que representa gastos médicos directos y los dos tercios restantes se deben a costos indirectos, tales como pérdida de productividad y ausentismo.

La mayoría de los casos de dolor lumbar crónico se clasifican y tratan como «lumbalgias inespecíficas», se consideran todos los casos que no tienen explicación por fracturas, traumatismos o por la presencia de enfermedades sistémicas, igualmente donde no existe compresión radicular demostrada. ${ }^{4}$

El tratamiento del dolor crónico lumbar incluye diferentes medidas como el uso de fármacos, la prescripción de ejercicio, en algunos casos reposo, el manejo de factores psicosociales relacionados y la corrección con factores posturales que pudieran condicionar la persistencia o recurrencia del dolor. ${ }^{5}$

Clínicamente, cualquier estrés anormal en la columna puede predisponer o precipitar el dolor de espalda baja. Una reducción de la lordosis lumbar ocasionada por tacones altos puede causar una fuerza anormal en los músculos, ligamentos y los huesos en esa región. ${ }^{6}$

El concepto de «postura» tiene múltiples acepciones; postura de pie es el resultado de un estado dinámico de equilibrio entre cuerpo y gravedad, una fuerza externa que atrae el cuerpo al suelo. Es la posición del cuerpo en el espacio y la disposición relativa de todas las partes del cuerpo que forman un acuerdo que establece una relación directa entre éste y la fuerza de gravedad. ${ }^{7}$

Aun en bipedestación estática existe un estado dinámico de equilibrio entre el cuerpo y las fuerzas de gravedad, que es mantenido por la contracción coordinada de los músculos antigravitatorios y la posición correcta de los segmentos corporales, lo que nos permite mantener el balance con un mínimo esfuerzo. ${ }^{8}$
Las fuerzas de gravedad se concentran en un punto específico del cuerpo conocido como «centro de gravedad», que en la posición anatómica y de manera general se localiza por delante de la segunda vértebra sacra, con variaciones discretas entre hombres y mujeres, debido a diferencias en la morfología pélvica. Este centro de gravedad puede variar de localización, puesto que depende de la disposición de las extremidades, la cabeza y el tronco. ${ }^{6}$

El uso de los zapatos de tacón se remonta a la población de origen indoeuropeo, que usaba dichos zapatos para fijar el pie en el estribo durante las marchas a caballo. Posteriormente la clase aristócrata inició su uso como símbolo de riqueza. Por último, el verdadero impulsor de este estilo tuvo lugar en Francia en 1670, cuando Luis XIV, llamado el Rey Sol, los usó para disimular su corta estatura. ${ }^{9}$

Previamente se sabía que al usar tacones el centro de gravedad se traslada a una posición posterosuperior que modifica la postura corporal. En el segmento lumbosacro y pélvico, el uso de tacones genera anteversión pélvica e hiperlordosis lumbar compensatoria; igualmente, los músculos paraespinales requieren reclutar más unidades motoras para mantener el balance. A largo plazo se reduce la flexibilidad de los músculos espinales y se produce distensión de los músculos abdominales, lo que causa desplazamiento anterior del centro de gravedad. ${ }^{6,8}$

Un zapato es un ítem de calzado fabricado para proveer comodidad y proteger el pie durante la vida diaria, existen varios tipos; sin embargo, durante el siguiente texto sólo se tomarán en cuenta los zapatos de tacón.

Dentro de las características importantes a valorar en el tacón resalta el ancho de éste, ya que el tacón grueso ayuda a distribuir la carga y asegura al usuario un mejor balance..$^{5,6}$

Desde hace ya más de cuatro décadas, existen publicaciones que reportan efectos negativos del uso de este tipo de calzado con lesiones musculoesqueléticas, como esguinces de tobillo y dolor de espalda baja, esto relacionado principalmente a las modificaciones biomecánicas posturales, derivado de ello se han establecido recomendaciones generales muy populares como restringir el calzado a un tacón «no superior a 3 centímetros». .0

Pero la literatura disponible sobre la asociación entre el uso de tacones y el riesgo de padecer dolor lumbar aún no es clara.

El objetivo del presente artículo fue analizar la información disponible relacionada con las alteraciones posturales de la columna vertebral asociadas al uso de tacones y, a su vez, al dolor lumbar. 


\section{MATERIAL Y METODOS}

Se realizó una revisión de la literatura disponible para dar respuesta a la incógnita de investigación utilizando los siguientes pasos: identificación de los motores de búsqueda, identificación de las palabras clave que permitan abordar el conocimiento, así como la selección y lectura de los estudios pertinentes.

Los estudios científicos se identificaron mediante búsqueda bibliográfica a través de las siguientes bases de datos: PubMed, EBSCO, ScienceDirect, Cochrane durante el periodo de 1990 a 2018.

Los términos de búsqueda fueron los siguientes: «lordosis lumbar, tacones/zapato de tacón, zapato alto, hiperlordosis y tacones/zapato de tacón, zapato alto, dolor lumbar y tacones/zapato de tacón, zapato alto, lumbalgia y tacones/zapato de tacón, zapato alto, columna y tacones/zapato de tacón, zapato alto, postura y tacones/zapato de tacón, zapato alto».

Se incluyeron artículos en inglés y español publicados entre 1994 y 2017, que describieran el uso de tacones de cualquier medida durante el estudio, tanto en población adolescente como adulta. Se excluyeron estudios realizados con plantillas o modificaciones al zapato diferentes al tacón.

Selección de estudios: se revisaron los títulos y los resúmenes de los artículos arrojados por las búsquedas. En primer lugar, con base en la revisión de títulos, se seleccionaron los que podrían ser elegibles 19; posteriormente, se procedió a leer cada uno de ellos, lo cual permitió descartar los artículos que no tenían relación con la pregunta de revisión. ${ }^{4}$
Esquema general de búsqueda. La búsqueda en las diferentes bases de datos permitió encontrar artículos; de éstos fueron descartados aquéllos que no cumplieran con alguno de los criterios de inclusión. Al término, los artículos seleccionados se utilizaron para el análisis final de la información (Figura 1).

\section{RESULTADOS}

Se ha postulado que los tacones causan alteraciones cualitativas en la mecánica de la marcha, afectan la cinética y cinemática de las estructuras corporales desde los pies hasta la columna, provocan dolor lumbar por incremento de la lordosis lumbar, existe un modelo sobre cómo se modifica la flexión plantar causando una compensación muscular que termina en un psoas hiperactivo, el cual desencadena hiperlordosis lumbar; sin embargo, se ha encontrado evidencia contradictoria con respecto al incremento de la lordosis lumbar, su asociación con dolor e incremento de altura en tacones. ${ }^{11}$

Esta pregunta no es reciente, existe información desde 1932 que inicia con Lateur y colaboradores, quienes encuentran resultados mixtos con varias pacientes que mostraron tanto aumento como disminución de la curvatura. ${ }^{12}$

Opila y su equipo ${ }^{8}$ detectaron retroversión pélvica y disminución de lordosis lumbar con uso de tacones, Franklin y colegas, Bendix y colaboradores reportaron que la lordosis lumbar y la anteversión pélvica disminuyen con el uso de tacones. ${ }^{6}$

Una posible explicación es que la altura del tacón no es un determinante en las alteraciones posturales

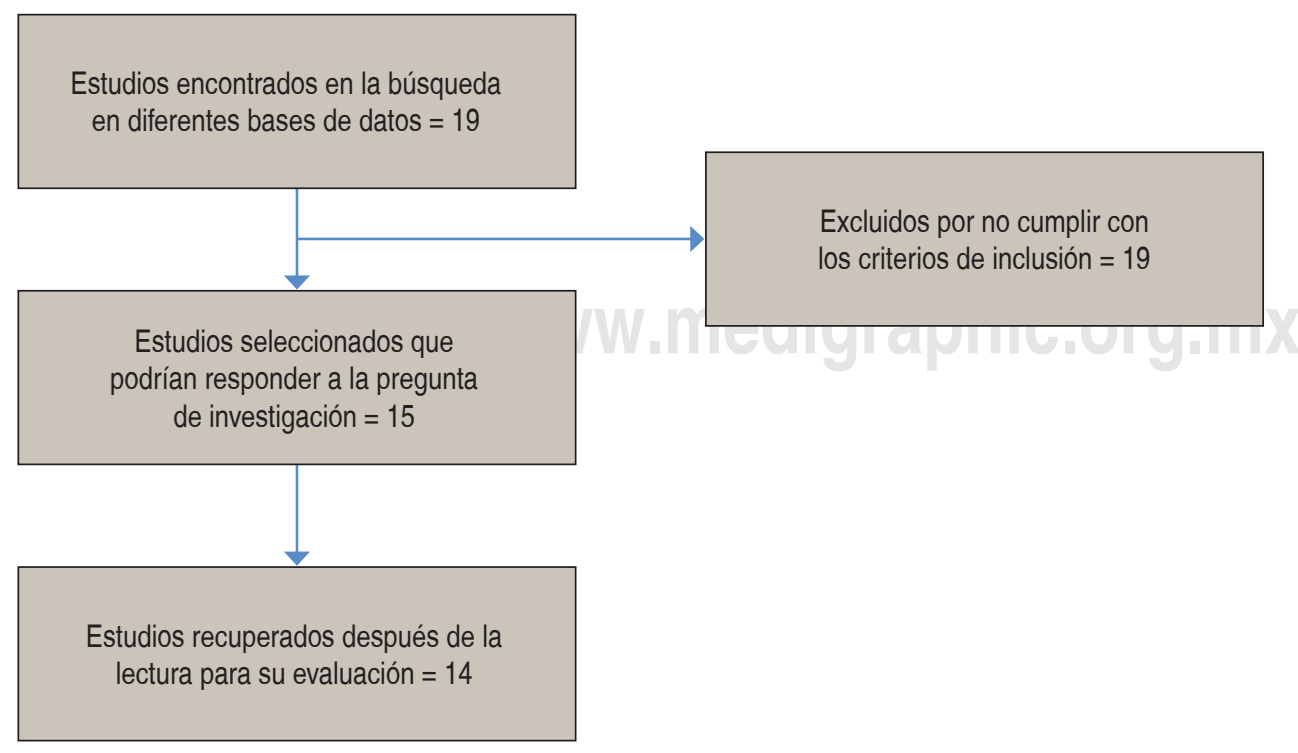

Figura 1:

Algoritmo que muestra la búsqueda y selección de artículos para la revisión. 
Tabla 1: Concentrado de información.

\begin{tabular}{|c|c|c|c|c|}
\hline Autor y año & Tipo de estudio & $\begin{array}{c}\text { No. } \\
\text { de pacientes }\end{array}$ & Intervención & Resultados \\
\hline Golightly YM, 2007 & $\begin{array}{l}\text { Autocontrolado expe- } \\
\text { rimental longitudinal ob- } \\
\text { servacional prospectivo }\end{array}$ & 12 & $\begin{array}{l}\text { Aumento } 3.18 \\
\text { por semanas sin } \\
\text { exceder } 9.54\end{array}$ & $\begin{array}{c}\text { Post: EVA general } 21.4 \pm 15.8 \text {, EVA bipedestación } 15.8 \pm 12 \\
\text { Oswestry } 10.2 \pm 5.0\end{array}$ \\
\hline $\begin{array}{l}\text { Dananberg HJ, } \\
1999\end{array}$ & $\begin{array}{l}\text { Autocontrolado expe- } \\
\text { rimental longitudinal ob- } \\
\text { servacional prospectivo }\end{array}$ & 32 & Ortesis a medida & $84 \%$ tuvo mejoría a $0.66 \%$ DE 0.78 \\
\hline Ferrari R, 2013 & Casos y controles & 28/32 & Ortesis & $\begin{array}{c}\text { Oswestry 16.9-8.8/26.4/1 10.9, discapacidad laboral 43.3/34., } \\
\text { prescripción de analgésicos } 60 / 31.3\end{array}$ \\
\hline MacRae CS, 2013 & Casos y controles & $58 / 57$ & $\begin{array}{l}\text { Zapato tipo flat/ } \\
\text { zapato tipo rocker }\end{array}$ & $\begin{array}{c}\text { Roland Morris 4.8/4.0, ENA 4.2/4.2, SFSS 7.1/6.7, EQ } \\
\text { 0.8/0.7, EVA HAD 75.6/70.5, escala de kinesiofobia de Tampa } \\
\text { 34.7/34.3, HAD [A] 6.0/6.3, [D] 3.5/4.3, satisfacción global } \\
33 / 17\end{array}$ \\
\hline Rosner AL, 2014 & Casos y controles & 19 & Ortesis/plantilla & $\begin{array}{c}\text { RM } 7.7 / 5.8, \text { NRS 5.3/2.7, FIX 5.0/3.9, } \\
\text { EVA: } 33 / 28 / 26 \text {, Oswestry: 30/26/24 }\end{array}$ \\
\hline Cambron JA, 2017 & ECA & 224 & $\begin{array}{l}\text { Ortesis a medida + } \\
\text { quiropráctico }\end{array}$ & \\
\hline Iqbal R, 2012 & $\begin{array}{l}\text { Autocontrol observacional, } \\
\text { longitudinal }\end{array}$ & 19 & & $\begin{array}{l}\text { Tacón 0: riesgo de patología lumbar } 16.79 \% \text {, } \\
\text { tacón 1: 19.0\%, tacón 2: 22\% }\end{array}$ \\
\hline Shabat S, 2005 & Prospectivo doble ciego & 58 & Placebo/plantillas & Placebo $79 \%$, plantilla $+30 \%$ \\
\hline $\begin{array}{l}\text { Castro-Méndez A, } \\
2013\end{array}$ & ECA & 51 & $\begin{array}{l}\text { Ortesis a medida/ } \\
\text { placebo }\end{array}$ & EVA 6.64/3.17, Oswestry 21-64/8.69 \\
\hline Larsen K, 2002 & ECA & 146 & $\begin{array}{l}\text { Plantillas } \\
\text { personalizadas }\end{array}$ & Sin resultados estadísticamente significativos. RR 0.7, ARR 19 \\
\hline Lisón JF, 2018 & ECA & 40 & $\begin{array}{l}\text { Zapatos inestables } \\
\text { por un periodo mí- } \\
\text { nimo de } 6 \text { horas al } \\
\text { día por } 4 \text { semanas }\end{array}$ & $\begin{array}{l}\text { Disminución en discapacidad con zapatos inestables comparados } \\
\text { con los controles }(-5,95 \%[\mathrm{Cl}]=-8.4 \text { a }-1.6) \text {. Incremento signifi- } \\
\text { cativo en el porcentaje de RA, ES, IO, y EO EMG, así como en el } \\
\text { ROM lumbar en zapatos inestables comparados con controles }\end{array}$ \\
\hline Schroeder J, 2018 & Crossover aleatorizado & 37 & Zapatos de tacón & $\begin{array}{l}\text { Sin cambios en el ángulo de lordosis lumbar } 0 \text { amplitud de } \\
\text { movimiento en condiciones estáticas o dinámicas }(p>0.05, d \\
\leq 0.06) \text {. Mínimo efecto en la reducción de inclinación pélvica } \\
(p=0.003, d=0.24) \text { efecto moderado para el incremento en } \\
\text { rotación pélvica }(p=0.001, d=0.63)\end{array}$ \\
\hline Elsayed W, 2017 & $\begin{array}{l}\text { Autocontrol observacio- } \\
\text { nal, longitudinal }\end{array}$ & 17 & $\begin{array}{c}\text { Zapatos con } \\
\text { diferente altura de } \\
\text { tacón }(0,6 \text { y } 10 \mathrm{~cm})\end{array}$ & $\begin{array}{c}\text { Sin diferencia significativa en EMG }(p=0.3 / 0.4) \text { de superficie } \\
\text { ni ángulo de lordosis lumbar }(p=0.54)\end{array}$ \\
\hline Vieira E, 2015 & ECA & 20 & $\begin{array}{l}\text { Durante la segundá } \\
\text { semana } 36 \text { horas } \\
\text { mínimo en un mes } \\
\text { uso de tacón }(161 \\
\mathrm{cm} / 165 \mathrm{~cm})\end{array}$ & $\begin{array}{l}\text { Sin cambios significativos en niveles de dolor o discapaci- } \\
\text { dad para el grupo control. El grupo con intervención reporta } \\
\text { menores niveles de dolor en la semana } 4 \text { (diferencia } \geq-1.4, p \\
\leq 0.009 \text { ) y } 6 \text { (diferencia } \geq-3.1, p<0.001 \text { ). Disminuyeron los } \\
\text { niveles de discapacidad en la semana } 4 \text { (diferencia }=-4.5 \% \text {, } \\
\text { p NS) y } 6 \text { (diferencia }=-14.1 \%, p=0.020 \text { ) }\end{array}$ \\
\hline
\end{tabular}

EVA = escala visual análoga, ECA = ensayo clínico aleatorizado, ENA = escala numérica análoga, $R O M=$ arco de movimiento lumbar, $E M G=$ electromiografía, $E S=$ erector espinal, $R A=$ recto abdominal, $I O=$ oblicuo interno, $E O=$ oblicuo externo.

de la columna, sino que los efectos de tacón alto presentan mayor relación con la costumbre al uso del zapato. $^{13}$

Schroeder y su equipo investigaron los efectos de zapatos de tacón con respecto a la posición pélvica (estática/dinámica) y lordosis lumbar en mujeres de mediana edad y con experiencia en el uso de este tipo de zapato; detectaron un efecto leve a moderado en la posición pélvica estática y dinámica; pero, no se observó relación entre el uso de zapatos de tacón y la hiperlordosis lumbar. ${ }^{14}$

Aun a la fecha la evidencia es controversial, solamente Oliveira y colegas ${ }^{15}$ encontraron una asociación entre el uso de zapatos de tacón y el incremento 
de la lordosis lumbar; no obstante, la población de estudio comprende sólo edades de 13 a 20 años, por tanto, podría deberse a la falta de experiencia que destaca como un factor relevante en los estudios previamente mencionados. ${ }^{8,16}$

El uso de zapatos de tacón puede causar una «reacción en cadena» para la transición espinopélvica, se concluye que las estrategias compensatorias posturales se presentan en la pelvis más que en la columna lumbar. Se ha observado un patrón de marcha alterado en mujeres jóvenes, es decir, un incremento en la rotación pélvica en el plano transversal; sin embargo, en mujeres de mediana edad no se han reportado hallazgos similares, lo cual hace énfasis en el rol de la habituación. ${ }^{17}$

Iqbal y colaboradores llevaron a cabo un estudio que incluyó a 19 estudiantes de universidad, caminando 50 metros con diferentes alturas de zapatos: sin tacón, zapatos con tacón de $1.54 \pm 0.84 \mathrm{~cm}$ y finalmente $5.50 \pm 1.70 \mathrm{~cm}$. Se midió la cinemática lumbar y se observó que los zapatos de tacón colocan al pie en una posición de plantiflexión, con una mayor cantidad de presión en el antepié. Esto ocasiona la necesidad de ajustar el resto del cuerpo para mantener el equilibrio donde la parte inferior del cuerpo se inclina hacia adelante, y el resto de los segmentos corporales se inclinan hacia atrás; este cambio en la postura puede ocasionar dolor lumbar y es más frecuente en mujeres con menos de $1.52 \mathrm{~cm}$ de estatura. ${ }^{18}$

El resumen de los estudios incluidos y los resultados obtenidos en relación con la presencia de dolor lumbar se muestran en la Tabla 1.

\section{DISCUSIÓN}

En resumen, la lordosis lumbar por lo general disminuye al aumentar la altura del tacón. No se ha encontrado una relación directa entre el nivel de experiencia en el uso de tacones y el movimiento pélvico. Como resultado, parece que la lumbalgia podría estar asociada con otros factores inducidos por los tacones altos diferentes al aumento o disminución de la lordosis lumbar.

Los zapatos de tacón son un ítem popular en la población, principalmente en adolescentes y adultos jóvenes. El dolor crónico lumbar es un problema de salud pública y a la fecha el tratamiento requiere de la intervención tanto en el manejo del dolor como en la prevención de recurrencias.

En nuestra revisión no se demostró que las alteraciones posturales de la columna se asociaran a hiperlordosis lumbar, por el contrario, la evidencia parece señalar mayores cambios en ajustes pélvicos con retroversión y cambios en la cinemática del patrón de marcha.

Es probable que la experiencia en el uso de calzado de este tipo sea el principal determinante en las alteraciones posturales; sin embargo, debido a las modificaciones cinemáticas de la marcha con el uso de tacones, lo más recomendable será usarlos de una altura adecuada para disminuir las alteraciones posturales. ${ }^{19}$

Con base en los resultados, la recomendación es el uso de un tacón entre 2.38 y $3.8 \mathrm{~cm}$, y evitar tacones que superen $7.2 \mathrm{~cm}$.

Será importante generar más estudios para identificar otros factores que pueden influir como el tiempo y tipo de uso, el peso de las pacientes, la presencia de dolor previo, la habituación a éstos, si poseen o no plataforma, la forma, el material, la cadencia, la superficie, así como el tipo de tacón.

En realidad existe poca literatura que evidencie la altura correcta del tacón para evitar alteraciones musculoesqueléticas. Por lo tanto, se requieren investigaciones más amplias para determinar con claridad el punto en el que la altura del tacón se vuelve biomecánicamente desventajosa y puede llegar a desencadenar dolor lumbar.

\section{CONCLUSIÓN}

El uso de tacones ocasiona alteraciones en la cinemática de la marcha y cambios posturales espinopélvicos con retroversión pélvica. Las alteraciones posturales parecen estar asociadas a la experiencia en el uso de este calzado.

El uso de tacones se relaciona en algunos artículos a reducción de la lordosis, retroversión pélvica y ajustes posturales que pueden asociarse a incrementar el estrés en los elementos posteriores del segmento lumbar y secundariamente contribuir al dolor lumbar.

Tanto la lordosis espinal como la cifosis dorsal disminuyen con el aumento de la altura del tacón; sin embargo, no se ha encontrado diferencia entre el nivel de experiencia para el movimiento espinal y pélvico.

Como resultado, parece que la lumbalgia podría estar asociada con otros factores inducidos por los tacones altos. El uso de tacones y la probabilidad de dolor de espalda baja dependen de la estatura del usuario, es decir, la estatura baja ha demostrado mayor riesgo de lumbalgia en comparación con tacones altos y mayor estatura. 


\section{BIBLIOGRAFÍA}

1. Smith BE, Littlewood C, May S. An update of stabilisation exercises for low back pain: a systematic review with metaanalysis. BMC Musculoskelet Disord. 2014; 15: 416.

2. Soto-Padilla M, Espinosa-Mendoza RL, Sandoval-García JP, Gómez-García F. Frecuencia de lumbalgia y su tratamiento en un hospital privado de la Ciudad de México. Acta Ortopédica Mexicana. 2015; 29 (1): 40-45.

3. Covarrubias-Gómez A. Lumbalgia: un problema de salud pública. Rev Mex Anest. 2010; 33 (S1): 106-109.

4. González-Maza C, Moscoso-López L, Ramírez-Elizalde G, AbdoAndrade A. Tratamiento multimodal para lumbalgia crónica inespecífica. Acta Ortopédica Mexicana. 2010; 24 (2): 88-94.

5. Diagnóstico, Tratamiento y Prevención de Lumbalgia Aguda y Crónica en el primer nivel de atención. México: Secretaría de Salud; 2009.

6. Franklin ME, Chenier TC, Brauninger L, Cook H, Harris S. Effect of positive heel inclination on posture. J Orthop Sports Phys Ther. 1995; 21 (2): 94-99.

7. Silva AM, de Siqueira GR, da Silva GA. Implications of highheeled shoes on body posture of adolescents. Rev Paul Pediatr. 2013; 31 (2): 265-271.

8. Opila KA, Wagner SS, Schiowitz S, Chen J. Postural alignment in barefoot and high heeled stance. Spine. 1988; 13 (5): 542-547.

9. Nieto E, Nahigian SH. Severe ankle injuries while wearing elevated "platform" shoes. Ohio State Med J. 1975; 71 (3): 137-141.

10. Linder M, Saltzman CL. A history of medical scientists on high heels. Int J Health Serv. 1998; 28 (2): 201-225.
11. Wiedemeijer MM, Otten E. Effects of high heeled shoes on gait. A review. Gait Posture. 2018; 61: 423-430.

12. de Lateur BJ, Giaconi RM, Questad K, Ko M, Lehmann JF. Footwear and posture. Compensatory strategies for heel height. Am J Phys Med Rehabil. 1991; 70 (5): 246-254.

13. Snow RE, Williams KR. High heeled shoes: their effect on center of mass position, posture, three-dimensional kinematics, rearfoot motion, and ground reaction forces. Arch Phys Med Rehabil. 1994; 75 (5): 568-576.

14. Schroeder J, Hollander K. Effects of high-heeled footwear on static and dynamic pelvis position and lumbar lordosis in experienced younger and middle-aged women. Gait Posture. 2018; 59: 53-57.

15. de Oliveira-Pezzan PA, João SM, Ribeiro AP, Manfio EF. Postural assessment of lumbar lordosis and pelvic alignment angles in adolescent users and nonusers of high-heeled shoes. J Manipulative Physiol Ther. 2011; 34 (9): 614-621.

16. Cronin NJ. The effects of high heeled shoes on female gait: a review. J Electromyogr Kinesiol. 2014; 24 (2): 258-263.

17. Mika A, Oleksy L, Mika P, Marchewka A, Clark BC. The effect of walking in high- and low-heeled shoes on erector spinae activity and pelvis kinematics during gait. Am J Phys Med Rehabil. 2012; 91 (5): 425-434.

18. Iqbal R, De A, Mishra W, Maulik S, Chandra AM. Study on lumbar kinematics and the risk of low back disorder in female university students by using shoes of different heel heights. Work. 2012; 41 Suppl 1: 2521-2526.

19. Baaklini E, Angst M, Schellenberg F, Hitz M, Schmid S, Tal A et al. High-heeled walking decreases lumbar lordosis. Gait Posture. 2017; 55: 12-14. 